\title{
Antica lirica italiana in catalano
}

\section{Costanzo Di Girolamo}

Università di Napoli «Federico II»

Lo strano titolo del quaderno di traduzioni dall'italiano di Miquel Desclot, Saps la terra on floreix el llimoner?, ${ }^{1}$ è preso in prestito dall' incipit di una poesia di Goethe, tradotta poi in esergo. Si tratta della canzoncina che a un certo punto del Wilhelm Meisters Lehrjahre (III, 1) l'enigmatica bambina Mignon canta al protagonista: benché nel romanzo non si dica da nessuna parte quale sia la terra dove fioriscono i limoni e dove le arance risplendono nell'ombra cupa del fogliame, molti hanno pensato, per ovvi motivi, a qualche località in Italia, piuttosto che in Turingia o in Pomerania. E così si spiega il titolo di questo libro, che raccoglie centosei traduzioni: da Dante (le quattro canzoni petrose), dal Canzoniere di Petrarca (ottantadue, tra canzoni, sonetti e ballate) e da Michelangelo (venti, tra sonetti, madrigali, epigrammi e frammenti).

Desclot rispetta in ogni dettaglio, con una fedeltà assoluta, la metrica e le rime dei modelli. A mo' di esempio, si legga la prima stanza della quarta petrosa: ${ }^{2}$

Così nel mio parlar voglio esser aspro com'è ne li atti questa bella petra, la quale ognora impetra maggior durezza e più natura cruda, e veste sua persona d'un diaspro tal che per lui, o perch'ella s'arretra, non esce di faretra

saetta che già mai la colga ignuda; ed ella ancide, e non val ch'om si chiuda né si dilunghi da’ colpi mortali,
Talment en els meus versos vull ser aspre com en els fets aquesta pedra bella, que sempre s'encastella amb més duresa i més natura crua,

$5 \mathrm{i}$ es guarneix la persona amb un diaspre tal que per ell, o perquè es retreu ella, del buirac no es dardella sageta que la pugui sobtar nua; i ella fereix, i no hi val girar cua 10 per allunyar-se dels seus cops mortals,

1. Miquel Desclot, Saps la terra on floreix el llimoner? Dante, Petrarca, Michelangelo, pròleg d'Anton M. EspaDAler, Barcelona: Proa [Els Llibres de l'Ossa Menor, 188], 1999, p. XVI172.

2. Il testo italiano non è a fronte di quello catalano. Le citazioni che seguono sono tratte dalle edizioni canoniche: Dante ALIGHIERI, Rime, a cura di Gianfranco ConTINI, Torino: Einaudi, 1965; Francesco Petrarca, Canzoniere, a cura di Gianfranco Contini, Torino: Einaudi, 1964; Michelangelo Buonarroti, Rime, a cura di Enzo Noè Girardi, Bari: Laterza, 1967. 
che, com'avesser ali,

giungono altrui e spezzan ciascun'arme: sì ch'io non so da lei né posso atarme. que, com ocells fatals, tot home atenyen i desfan tota arma: tal que d'ella no sé com defensar-me.

Chiunque, credo, è in grado di apprezzare l'eccezionalità di questo risultato, che, con i prevedibili alti e bassi, si estende a tutta la raccolta, sostanzialmente omogenea per qualità e efficacia. Desclot propone delle esatte repliche, in un'altra lingua, degli originali, sicché il lettore catalano, pur sentendosi a casa sua, può aggirarsi all'interno di forme metriche complesse e grandiose, e in buona parte ignote alla sua tradizione medievale. Si noti con attenzione il procedimento del rimamento di questa stanza. Alcune delle rime dantesche sono conservate, con le ovvie diversità fonetiche nel passaggio da una lingua all'altra: -aspro >-aspre, -uda > -ua, -ali >-als, -arme >-armal-arme. Ma, insieme con la rima, sono in alcuni casi conservati anche i rimanti, cioè le parole in rima, con i significati che si portano appresso: aspro > aspre, diaspro $>$ diaspre, cruda $>$ crua, nuda $>$ nua, mortali $>$ mortals, arme $>$ arma, atarme $>$ defensar-me (qui diversa la forma, ma identico il significato); in altri casi no: in cua e fatals i rimanti cambiano completamente rispetto a chiuda e a ali. L'altra rima della stanza, che ricorre quattro volte (le altre rime ricorrono tre e due volte), è -etra, rima aspra associata al rimante, che è poi la parola-chiave dell'intero ciclo, petra: qui il traduttore sembra gettare le armi e sceglie una rima facile oltre che dolce, -ella. È chiaro che quando, oltre alle rime, passano anche i rimanti, i significati complessivi dell'originale vengono meglio conservati o riprodotti con totale precisione; ma non è detto che quando si cambia di rimante o addirittura di rima avvenga sempre il contrario. Al v. 3, impetra significa probabilmente "racchiude come in pietra» (o, almeno, Desclot ha preferito questa interpretazione a un'altra pure possibile, 'vuole con fermezza'), sicché encastella rende assai bene il verbo italiano; nessun cambio, ma semplici inversioni, anche ai vv. 2, 6 e 7. Al v. 9, invece, c'è un deciso impoverimento semantico, laddove si perde ch'om si chiuda "che ci si corazzi» e resta solo l'idea della fuga; al v. 11, infine, viene concretizzata fino all'eccesso un tenue spunto metaforico: i colpi veloci (veloci com'avesser ali) diventano senz'altro degli ocells fatals, dando inopinatamente vita a un'immagine alla Hitchcock.

Il lettore italiano troverà che questa lingua calza perfettamente sui suoi antichi poeti, con una straordinaria versatilità. Il Dante delle petrose volto in catalano, una lingua molto vicina all'antico occitano, sembra quasi miracolosamente restituito alla lingua stessa di Arnaut Daniel, l'ideale e onnipresente modello del ciclo. D'altro canto, Petrarca è reso con levigata dolcezza. ${ }^{3}$ Si veda

3. Le prime traduzioni in catalano dal Canzoniere risalgono alla fine del diciannovesimo secolo, ma l'opera non è mai stata tradotta per intero. La selezione più estesa si deve a Osvald CARDOna, F.P., Sonets, cançons $i$ madrigals, Barcelona: Alpha, 1955; a cui seguì quella di Francesc DE RIART, F.P., Un Centenar de sonets a Laura, Barcelona: El traductor, 1968. Sulle versioni, degli anni venti, di Agustí EsCLASANS e di Maria-Antònia SALVÀ, si veda lo studio di Gabriella GAVAGNIN, "Note su alcune traduzioni catalane novecentesche di Petrarca», La parola del testo, III (1999), p. 381-402. 
ad esempio la prima stanza di Chiare, fresche et dolci acque (dove però, curiosamente, il seno di Laura diventa un respiro):

Chiare, fresche et dolci acque, ove le belle membra pose colei che sola a me par donna; gentil ramo ove piacque (con sospir' mi rimembra) a lei di fare al bel fianco colonna; herba et fior' che la gonna

leggiadra ricoverse co l'angelico seno, aere sacro, sereno, ove Amor co' begli occhi il cor m'aperse: date udienza insieme a le dolenti mie parole estreme.
Fresca i dolça aigua clara, on els bellíssims membres posava la qui tinc per sola dona; gentil branca on encara

5 (ah, Déu, com m’ho remembres!) el dolç frec del seu flanc cansat ressona; herba i flors que la bona gonella recobria

10 i l'angèlic alè; aire sacre, serè, on Amor ab bells ulls el cor m'obria, doneu oïda ensems, als meus adolorits dictats extrems.

Quanto poi al rude dettato di Michelangelo, spesso involuto e raziocinante, attento più alla descrizione ossessiva delle 'cose concrete' che all'evocazione di situazioni e di immagini, non può non ricordare, tanto più messo in catalano, quello di Ausiàs March, con cui presenta non poche affinità. Si leggano queste due quartine epigrammatiche:

II

Sol io ardendo all'ombra mi rimango, quand'el sol de' suoi razzi el mondo spoglia:

ogni altro per piacer, e io per doglia, prostrato in terra, mi lamento e piango.
Jo sol cremant a l'ombra m'arroento quan dels seus raigs despulla el món el sol: tots els altres per goig, i jo per dol prostrat a terra, em planyo i em lamento.

\section{LXIV}

Se 'l foco il sasso rompe e 'l ferro squaglia, Si el foc la pedra romp i el ferro talla, figlio del lor medesmo e duro interno, che farà 'l più ardente de l'inferno d'un nimico covon secco di paglia? fill com és del seu cor mateix intern, ¿què farà el més ardent de tot l'infern amb una garba de resseca palla?

Il quaderno di traduzioni di Desclot si presenta dunque come un vero capolavoro di bravura da parte di un traduttore competente che padroneggia molto bene la lingua di entrata e si rivela un consumato verseggiatore in quella di uscita. Lodi maggiori io non riuscirei a farne: chi più ne ha, più ne metta, se lo ritiene opportuno. Tuttavia, un libro come questo pone secondo me degli inquietanti interrogativi sul significato e sulle finalità delle traduzioni dei testi poetici. La traduzione di un autore antico, nei confronti del quale esista una differenzialità, oltre che, ovviamente, linguistica, anche culturale (significati pregnanti di alcune parole, riferimenti a universi ideologici non più attuali, ecc.) e formale (la metrica, ma anche i livelli stilistici, ecc.), può semplicemente 
mirare a renderlo comprensibile e a ridargli vita presso un nuovo pubblico: sono queste le traduzioni cosiddette filologiche. La loro bellezza e efficacia può variare a seconda delle capacità del traduttore: non devono essere necessariamente servili, ancillari, né, come si dice, letterali (nessuna traduzione può esserlo veramente); ma è evidente che, se questo è il loro fine, chi traduce deve astenersi dall'imporre la sua impronta sui significati del testo, cambiandoli o deformandoli. Esiste poi la traduzione-rifacimento o la traduzione-parodia: un'idea di Brecht, ripresa una trentina di anni fa da Fortini, e di cui ho parlato di recente a proposito delle traduzioni catalane della Divina Commedia. ${ }^{4}$ Secondo Brecht, queste traduzioni sono da preferire alle false traduzioni d'arte, che servono semplicemente a imbellettare un testo in vista della sua mercificazione; e forse è vero che un certo tipo di opere (penso in particolare al teatro o a romanzi di grande complessità linguistica) solo così può essere tradotto, stabilendo cioè una voluta distanza dal testo. Ed esistono infine le vere traduzioni d'arte: nel caso della poesia, poeti che traducono altri poeti, o più semplicemente traduttori che si rivelano veri poeti, anche se non lo sono in proprio, nell'atto stesso della traduzione. Quali caratteristiche dovrebbe avere questo tipo di traduzioni? Anche qui, suppongo, il traduttore dovrebbe frapporre un minimo di distanza tra sé e il testo, certo non la distanza della parodia e del rifacimento, ma, questo sì, prendere atto della differenzialità di cui si parlava prima e, arrogandosi una certa libertà, assumersi per intero determinate responsabilità. Il punto d'arrivo della traduzione d'arte di una poesia è quello di dare vita a un'altra poesia, che rispetto all'originale avrà inevitabilmente un suo deficit in un aspetto $\mathrm{o}$ in un altro, ma che potrà essere apprezzata come un'opera autonoma, di per sé valida (gradino a cui la traduzione filologica non può quasi mai arrivare). Da questo punto di vista, la traduzione d'arte svolge un ruolo propulsore anche all'interno delle singole tradizioni letterarie. Tutti sappiamo che la letteratura italiana nasce con una traduzione, quale sostanzialmente è Madonna, dir vo voglio di Giacomo da Lentini, basata da vicino su una canzone di Folchetto di Marsiglia; e traduttori sono stati un numero incalcolabile di scrittori, fino al caso del secolo XX, quando la traduzione d'arte è diventata per molti come una prova obbligata, un passaggio ineludibile. Tutto questo si capisce facilmente, e dovrebbe essere condivisibile da tutti. Da tutti, voglio dire, dovrebbe essere condivisa l'idea che la poesia in uscita dovrebbe essere leggibile come una 'normale' poesia di un poeta contemporaneo. Si leggano ad esempio questi versi:

Spesso, a lusingar vette, vidi splendere sovranamente l'occhio del mattino, e baciar d'oro verdi prati, accendere pallidi rivi d'alchimìe divine.

Poi vili fumi alzarsi, intorbidata

4. «La Divina Comèdia en català», in L'Espill, n. 7 (2001), p. 131-140. Il saggio di Franco FORTINI è «Traduzione e rifacimento» (1972), nel volume Saggi italiani, Milano: Garzanti, 1987, p. 359-379. 
d'un tratto quella celestiale fronte, e fuggendo a occidente il desolato mondo, l'astro celare il viso e l'onta.

Anch'io sul far del giorno ebbi il mio sole e il suo trionfo mi brillò sul ciglio: ma, ahimè, poté restarvi un'ora sola, rapito dalle nubi in cui s'impiglia.

Pur non ne ho sdegno: bene può un terrestre sole abbuiarsi, se è così il celeste.

Chi abbia la fortuna, per così dire, di non conoscere o non ricordare questa poesia, può divertirsi per un momento, senza leggere oltre il prossimo punto, a indovinare chi ne è l'autore, a tentare un'approssimativa datazione, a interpretare la metrica. La lingua, che risente ancora di qualche tratto dannunziano, dovrebbe portarci, grosso modo, intorno ai decenni centrali del Novecento; le rime, quasi tutte irregolari, hanno uno schema ABAB CDCD EFEF GG, che è quello del sonetto elisabettiano (per complicare un po' le cose, ho eliminato solo il rientro tipografico che nell'originale hanno gli ultimi due versi). A questo punto, un lettore sufficientemente competente della poesia italiana del Novecento dovrebbe pensare senza difficoltà a Montale: la poesia suona come perfettamente montaliana e una puntuale analisi linguistica e metrica potrebbe confermarlo. Si tratta della traduzione del sonetto XXXIII di Shakespeare, pubblicata per la prima volta nel 1944 (anche se l'autore ci informa in una nota che è anteriore al '38): ${ }^{\text {' }}$

Full many a glorious morning have I seen Flatter the mountain tops with sovereign eye,

Kissing with golden face the meadows green,

Gilding pale streams with heavenly alchemy;

Anon permit the basest clouds to ride

With ugly rack on his celestial face,

And from the forlorn world his visage hide,

Stealing unseen to west with this disgrace:

Even so my sun one early morn did shine

With all triumphant splendour on my brow;

But out, alack, he was but one hour mine,

The region cloud hath masked him from me now.

Yet him for this my love no whit disdaineth;

Suns of the world may stain when heaven's sun staineth. ${ }^{6}$

5. Eugenio MONTALE, L'opera in versi, edizione critica a cura di Rosanna BETTARINI e Gianfranco ConTINI, Torino: Einaudi, 1980, p. 712 (note alle p. 1154-55).

6. William ShaKesPeare, The Sonnets and A Lover's Complaint, edited by John KerRigan, Harmondsworth: Penguin Books, 1986, p. 93. Sia detto per inciso che questo sonetto, dall'interpretazione molto controversa, è secondo me un'alba: vedi in particolare i vv. 9-12. Com'è noto, il genere dell'alba descrive o allude all'incontro o alla separazione degli amanti alle prime luci del giorno; sembra essere un archetipo della lirica, talvolta incastonato in 
Montale, in effetti, riprende la forma dell'originale (già importata nel continente da Mallarmé, che non a caso era un professore di inglese), ma vi introduce un tipo di rime tipiche della sua produzione, o comunque della poesia italiana di quegli anni, sicché essa non si presenta come un anacronismo né come un esotismo. Per di più, quella del sonetto elisabettiano appare come una forma produttiva in Montale, probabilmente anche perché consiste di tre quartine, una misura cara al poeta, seguite da un distico: nella Bufera compaiono ben quattro sonetti simili, tra cui i famosissimi Orecchini, tutti degli anni 1940-42, di modo che, almeno come data di pubblicazione, essi precedono i tre sonetti di Shakespeare, apparsi tra il '44 e il '47. La traduzione di Montale è dunque una «normale» poesia: se se ne ignorasse la provenienza, e in assenza di riferimenti rivelatori, potrebbe giustamente essere scambiata per una poesia scritta direttamente in quella lingua, in quegli anni precisi.

Il che è esattamente quanto non si verifica nelle meravigliose versioni di Miquel Desclot, che usa delle forme metriche, come la canzone all'italiana, che non hanno alcun precedente nella tradizione catalana e la cui complessa struttura, certo più complessa di un sonetto elisabettiano e ovviamente di un haiku, appare del tutto incomprensibile se avulsa dal suo contesto culturale. Con questo non voglio dire che, traducendo, non ci si possa porre come obiettivo l'approssimazione a tutti gli aspetti, compresi quelli più specifici, del testofonte e il loro trasferimento in una nuova lingua; né che il traduttore debba ad ogni costo attualizzare ciò che traduce (un pessimo esempio, al riguardo, sono le traduzioni di Guido Ceronetti da Catullo e Marziale). Non di meno, pur volendo ammettere che un'operazione di archeologia letteraria o di immedesimazione totale in un altro universo ideologico e formale sia in circostanze particolari giustificabile, essa non andava perseguita a danno del senso e in nome di valori tutt'altro che assoluti. Infatti, il rispetto sacrale per la rima perfetta e per il sillabismo rigoroso è ancora così osservato ed è così irrinunciabile? Sono domande che pongo ma, in ogni caso, io credo che nessun lettore potrebbe seriamente prendere queste versioni come poesie catalane contemporanee, databili agli ultimi anni del secolo scorso, né tanto meno come una ricostruzione, filologicamente solida, delle forme e, insieme, dei significati degli originali. Queste belle traduzioni sono in realtà dei falsi letterari, o forse qualcosa di peggio: se un falso è un'opera spacciata per antica, in questo caso nemmeno ciò avviene, perché queste versioni non sono collocabili in nessun tempo della tradizione della lingua di uscita. Dovrebbe essere perciò chiaro che il bravissimo Desclot ha dato al lettore catalano non qualcosa di più rispetto a quanto gli veniva chiesto, ma qualcosa di diverso; e questo lettore potrebbe anche avere ragione ad arrabbiarsi se si accorgesse che, per raccapezzare una

altri generi (come in Romeo and Juliet, atto III, scena V, dove, significativamente, ricorrono alcune immagini e metafore qui adottate), ed è presente in tutte le culture letterarie e in tutte le epoche (si ricordi il classico libro di Arthur T. HATTO, Eos: An enquiry into the theme of lovers' meetings and partings at dawn in poetry, The Hague: Mouton, 1965). 
rima e per non rinunciare all'impeccabilità dell'esercitazione metrica, si è attribuita a Dante un'immagine così ridicola e assurda come quella degli uccelli fatali e si è fatto intendere che Petrarca, avendo visto Laura nuda al bagno, ne elogiasse il respiro e non il seno. 